\title{
Reconfigurable RF Components for Multifunction RF Systems
}

\author{
Stephen Hary ${ }^{1}$, Taylor Barton ${ }^{2}$, John Ebel $^{1}$ \\ ${ }^{1}$ Air Force Research Laboratory, Dayton, OH, 45433, email: stephen.hary@us.af.mil, \\ ${ }^{2}$ University of Colorado Boulder, Boulder, CO, 80309
}

\begin{abstract}
The proliferation of the wireless market has driven the demand for RF systems to dramatically increase functionality, frequency coverage, bandwidth, and efficiency; while simultaneously reducing power consumption and die size to save power and cost. For example, the highly complex RF systems in handset technology have seen a dramatic increase in frequency, operating bands, and complexity of signals with each generation (from $1 G$ to $4 G$ ). The next generation (5G) will continue the trend to extend the frequency in to $\mathrm{mm}$-waves due primarily to spectrum congestion, bandwidth, and data rate. DoD is facing comparable challenges in terms of increasing the frequency of operation, bandwidth, and functionality while maintaining cost and accelerating technology insertion (or time to market for commercial). This paper reports on the impact of these new challenges for RF components and discusses solutions under investigation.
\end{abstract}

Index Terms - Reconfigurable architectures, Tunable circuits and devices, Cognitive radio, Scalability, Switched circuits

\section{INTRODUCTION}

Wireless System and sensor technology requirements increase dramatically with each generation upgrade for both commercial and military applications. New system demands for increasing functionalities (multi-mode and multi-standards) along with backward compatibility while simultaneously reducing system cost, size, weight, and power resulting in tremendous challenges for RF designers.

Traditional RF microsystems use fixed-frequency transceivers optimized for performance in a particular fixed band of interest. With the rapid increase in electromagnetic spectrum (EMS) utilization, these fixed frequency systems are increasingly vulnerable to in band and near band interference. The trend in commercial wireless is to design highly optimized compact chipsets for each operating band with each generation seeing a dramatic increase in frequency, operating bands, and RF performance. The trend for military systems is to push the digital interface closer to the RF front-end and perform programmable and reconfigurable functions in the digital domain where programmable signal processing can be accomplished with CMOS-based digital signal processing
(DSP). This approach works best if a wide instantaneous bandwidth is digitized, however maintaining high dynamic range and efficiency over broad instantaneous bandwidths is challenging. Performance of the overall transceiver and optimization of the DSP blocks can be enhanced by a reconfigurable RF front end. However, this type of reconfiguration has typically come with unacceptable performance penalties in the form of switching loss, reduced gain, reduced efficiency and increased spurious transmissions.

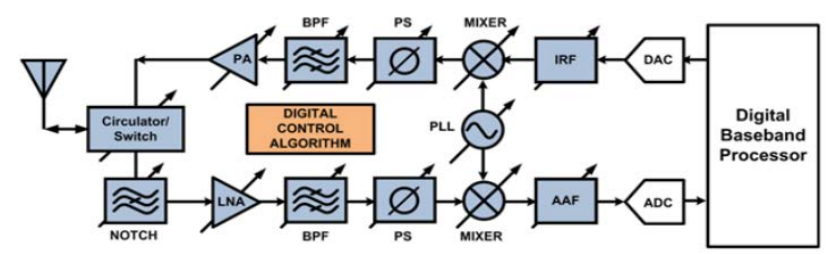

Fig. 1. Reconfigurable Transceiver Block Diagram

Fig. 1 shows a notional reconfigurable transceiver architecture. Similar to a traditional RF frontend (RFFE), it consists of a transmitter and a receiver to enable radar or communication function. The RFFE is further divided into transmit / receive (T/R) module and receiver/exciter module. Critical blocks for the $\mathrm{T} / \mathrm{R}$ module are the power amplifier (PA), low noise amplifier, RF switch, and phase shifter (for phased-array technology). The receiver/exciter module consists primarily of mixers, filters, and variable gain buffer amplifiers. Salient features of the reconfigurable architecture include the ability to 1) adjust frequency plan, both RF and IF, 2) adaptively filtering to enhance signals of interest and reject unwanted interfering signals, 3) adjust gain to optimize system dynamic range and efficiency, 4) adjust instantaneous bandwidth.

In order to realize the architecture depicted in Fig. 1, several reconfigurable components and enabling technologies are needed. Fortunately, there have been recent advances in key RF blocks and enabling technologies to make a reconfigurable RF system. This paper examines drivers to explore innovative solutions to meet the demands of future RF systems by implementing reconfigurable RF components. This paper first examines the fundamental building block, the RF switch, to enable 
reconfigurable RF components. A comparison of critical switch parameters is assessed for various RF switch technologies: solid state switches, MEMS-based switches, and phase change material (PCM) switches. The next section surveys PA techniques allowing multi-mode operation. The final section describes reconfigurable receiver/exciter architectures for efficient frequency conversion.

\section{RF SWITCH TECHNOLOGIES}

For reconfigurable RF components, a low loss, high frequency, and high power handling RF switch is a fundamental building block to tune the input/output matching networks for adjusting impedance states to match the active devices. RF switch technology is divided into three categories: solid state switches, microwave electromechanical switches (MEMS), and phase change material (PCM) switches. This section examines these switch technologies with respect to (1) insertion loss, (2) isolation, (3) power handling, and (4) switching speed.

\section{A. Solid State RF Switches}

There are a variety of solid state RF switches available on the market. These range from the low power silicon $\mathrm{CMOS} / \mathrm{SiGe}$ to the high power handling in $\mathrm{GaN}$ technology. A salient feature of the solid state switches is the fast switching speed, typically in 10s of nano-seconds. The main differences between these switches are insertion loss and power handling. Gallium-nitride $(\mathrm{GaN})$ highelectron mobility transistors (HEMT) switches are an attractive technology because they can potentially be monolithically integrated with state-of-the-art GaN HEMT microwave power amplifiers. Broadband (DC to $18 \mathrm{GHz}$ ) GaN HEMT switches are commercially available with insertion loss below $1.5 \mathrm{~dB}, 30 \mathrm{~dB}$ isolation, $10 \mathrm{~W}$ power handling, and $35 \mathrm{~ns}$ switching speed. Silicon and GaAs PIN (p-type intrinsic n-type) diodes have been a reliable, mature high-performance RF switching technology for many years. PIN switches dissipate significantly more power than FET switches. Broadband $(0.050-26.5 \mathrm{GHz})$ silicon PIN switches are commercially available with insertion loss below $1 \mathrm{~dB}$, isolation greater than $50 \mathrm{~dB}, 30$ $\mathrm{dBm}$ power handling, linearity greater than $40 \mathrm{dBm}$, while dissipating approximately $50 \mathrm{~mW}$, in both the on-state and off-state for a series-shunt configuration. Broadband $(0.2$ - $18 \mathrm{GHz}$ ) GaAs PIN switches are commercially available with insertion loss below $0.5 \mathrm{~dB}$, isolation greater than 35 $\mathrm{dB}$, while dissipating approximately $20 \mathrm{~mW}$, in both the on-state and off-state for a series-shunt configuration. Silicon-on-insulator (SOI) field effect transistors (FETs) have increased in RF performance over the years. While SOI switches still cannot realistically compete with $\mathrm{GaN}$ in the areas of bandwidth and power handling; they have significantly better insertion loss than CMOS switches and have replaced GaAs pHEMT in many wireless front-end applications requiring large number of RF switch ports.

Another GaN device, the super lattice castellated gate FET (SLCFET) [1], is a variant of GaN HEMT technology currently under research and development. Preliminary laboratory results have shown excellent RF performance, potentially capable out-performing all other types of solid-state switches. The SLCFET cannot be easily monolithically integrated with a conventional GaN HEMT microwave amplifier process due to different material profile. Manufacturability and lifetime of SLCFET switches have not yet been established.

\section{B. Phase-Change Switches}

Another type of solid-state switch made from thin-film germanium-telluride $(\mathrm{GeTe})$ is in the early stages of research and development at a handful of laboratories. GeTe switches operate by changing between polycrystalline and amorphous solid phases through a pulsed heating process. Although good RF performance has been demonstrated, currently the lifetimes of these devices are well short of what is required for a T/R switch. Switching times are in the 100-200 ns range.

\section{Microwave Electromechanical Switches}

RF microelectromechanical systems (MEMS) switches have demonstrated exceptional RF performance but suffer from slow switching speeds (5-10 microseconds) and present challenges in the areas of packaging, integration, and manufacturing. Although many groups have demonstrated high-performance RF MEMS switches, there are currently no commercial off-the-shelf sources for these devices.

\section{RECONFIGURABLE POWER AMPLIFIERS}

Multi-function, multi-mode T/R modules require PAs able to operate on signals both with different waveform types (instantaneous bandwidth, peak-to-average power ratio, etc) and at different frequencies. While efficiencyenhancement techniques such as Doherty and envelope tracking (ET) provide improved efficiency for high-PAPR signals, and wideband PAs are capable of covering multiple bands, developing a PA solution that is simultaneously linear, efficient, and wideband remains an active challenge. In this section, we summarize a sample of approaches based on reconfigurable structures to beat the linearity-efficiency-bandwidth tradeoff of conventional PAs.

\section{A. Waveform-Agile PAs}

Waveform-agile PAs allow the active devices to operate efficiently at varying power levels or signal PAPRs, maintaining favorable conditions through techniques 
including adaptive bias [2], reconfigurable output [3] or interstage [4] matching networks, or combinations of these techniques [5]. Architectures such as Doherty and ET PAs can also support waveform-agility, particularly when made reconfigurable through bias control $[2,6]$.

Although primarily used in CMOS PAs [4,5], power scaling through reconfigurable device periphery has been demonstrated at AFRL in GaN. Theoretically, increasing the device periphery by $2 \mathrm{x}$ will increase output power by 3 $\mathrm{dB}$, and when combined with drain supply variation, a total of $10 \mathrm{~dB}$ power scaling is achievable. Analysis shows that placing the RF switch at the gate terminal results in reduced loss compared to the drain, largely because the switch insertion loss is on the input side of the device. A reconfigurable transistor core was fabricated in a $0.25 \mu \mathrm{m}$ $\mathrm{GaN}$ foundry process and measured with device on/off states and power supply scaling as summarized in Table 1.

Table 1. Load Pull measured data of the reconfigurable transistor core at $3 \mathrm{GHz}$.

\begin{tabular}{|l|l|l|l|}
\hline Pin (dBm) & VDD (V) & Pout (dBm) & PAE (\%) \\
\hline One device on & 10 & 27.2 & 49 \\
\hline 21.7 & 20 & 29.7 & 53.3 \\
\hline 21.7 & 30 & 31 & 53 \\
\hline 21.7 & 10 & 29.6 & 50.1 \\
\hline \multicolumn{5}{|l}{} \\
\hline 24.6 & 20 & 32.9 & 53.6 \\
\hline 24.6 & 30 & 34.2 & 63 \\
\hline 24.6 & &
\end{tabular}

\section{B. Frequency-Reconfigurable PAs}

Traditionally, multiband RF frontends have been implemented using either PAs with fixed matching networks covering a broad frequency, or by using multiple individual narrowband PAs. An ideal solution for a true multi-function, multi-mode T/R module, however, would use a single PA operating at an arbitrary RF carrier.

Of particular interest are those techniques that incorporate waveform-agile efficiency enhancement architectures in a multi-band PA. Techniques incorporating power combining, such as Doherty and outphasing PAs, can be difficult to extend to wideband operation while maintaining loading conditions on the device. Nonetheless, significant effort has been made to develop wideband Doherty techniques. One example with both extended bandwidth and reconfigurable efficiency is found in [6], in which a modified Doherty structure is reconfigured using the relative phase of its RF inputs (rather than tunable reactive elements) and operates from 1.5 to $2.5 \mathrm{GHz}$ with over $49 \%$ drain efficiency.

Alternatively, direct dynamic load modulation (DLM) provides load modulation as in a Doherty amplifier, but by directly controlling the load impedance through a tunable OMN. Although it lacks the $\lambda / 4$ impedance inverter that limits conventional Doherty bandwidth, DLM is typically designed for single-band operation due to the challenges of the DLM matching. Despite this, an octave-bandwidth adaptive PA based on a commercial GaN HEMT and diode varactor tuning has been demonstrated with $64 \%$ $79 \%$ peak-power efficiency over 1-1.9 GHz [7].

Limitations in power handling of varactors and RF switches can present limitations in tunable networks for high-power applications. An alternative technique that employs phase control rather than tuning of circuit elements has recently been reported [8]. This load modulated balanced amplifier approach uses active drive of the isolation port to control the load impedance trajectory for the branch PAs over a broad frequency range. The approach has been shown over $800-2000 \mathrm{MHz}$ with $>70 \%$ efficiency.

\section{RECEIVER/EXCITER ARCHITECTURES}

At the system level, RF switches may be employed to enable coarse-grained, mid-grained, or fine-grained reconfigurable RF components and systems as depicted in Fig 2. Coarse grain architecture employs RF switches to select different components or channels such as fixed frequency/function high band, mid-band, or low band RF blocks. Medium grain architectures can tune the input/output matching networks for adjusting impedance states to active devices, altering the transfer function of specific devices. Fine grain architecture consists of a matrix of RF switches to connect both active and passive elements to form an RF function.

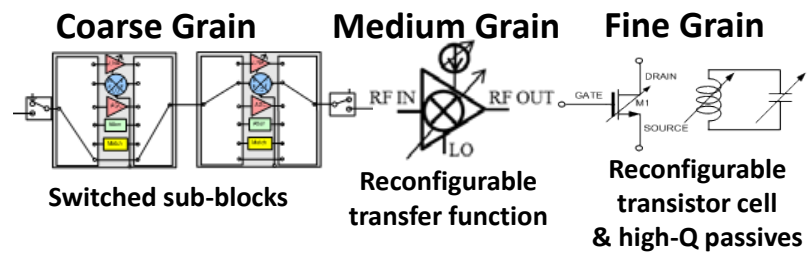

Fig 2: Granularity of reconfigurable architectures

An example of a coarse grain reconfigurable component is the ubiquitous switched filter bank, where $\mathrm{N}$ unique filters are laid out in parallel with single pole $\mathrm{N}$ throw switches on either side to create an $\mathrm{N}$-way switched filter bank. Similarly, the entire RF chain could be constructed from switched fixed function components/blocks. 
However, this implementation leads to size and cost that increases linearly with number of possible configurations. Fine grain architectures are not yet practical solution due to unacceptable performance penalties associated with reconfigurable transistor cells with a large numbers of cells.

Reconfigurable PAs discussed in this paper are examples of a medium grain reconfigurable component. Another example are tunable filters based on tuned cavities or compact capacitively coupled resonators $[9,10]$. These filters can be made more compact, and with greater tuning fidelity than coarse grain switched filter banks.

The heart of a medium grain reconfigurable architecture are reconfigurable agile core components depicted in Fig 2. These "supercomponents" can transform operation from variable gain amplifier, to mixer to through-line depending on bias and drive settings. Wideband programmable linearity, gain and power consumption are key attributes to make the supercomponent viable. SiGe is a leading candidate technology to make supercomponents with InP offering potentially lower NF and greater linearity at the expense for greater cost and lower levels of integration.

The MATRICs chip is an example of a medium grain transceiver built by integrating medium grain configurable $\mathrm{RF} /$ baseband block and microwave blocks [11]. This architecture is enabled by advances in $\mathrm{SiGe}$ switch technology.

Another key attribute of reconfigurable RF systems is granularity of reconfiguration. Options include: Factory reconfigurable: where connections and bias/passive settings are tailored for each application but cannot be changed, resulting in lower NRE and design time than full custom solutions, Reconfigure at system start: where a configuration is loaded at system startup but cannot be changed while running, Reconfigure mode-to-mode: where the user can initiated configuration changes (open loop), Reconfigure on-the-fly to predefined states: where the system can sense and reconfigure autonomously to a limited set of predefined states (closed loop), or Reconfigure on-the-fly, where the system can sense and reconfigure autonomously to any legal state (cognitive closed loop). The time constant associated with reconfiguring and settling varies dramatically for each level of granularity from weeks for factory reconfiguring to seconds for reconfigure at startup to msec for open loop and usec for closed loop reconfiguration. The options discussed in this paper are most suited to reconfigure mode-to-mode. On the fly reconfiguration requires decision logic embedded with the RF control which is not addressed in this paper

Building on these ideas, the Air Force Research Laboratory has initiated a research effort to demonstrate leap-ahead agile RF front-end systems with multi-function transmit / receive technology by employing reconfigurable RF \& mixed-signal components [12].

\section{CONCLUSION}

In this paper, we discuss key attributes of a reconfigurable RF system and highlight current state of the art for critical reconfigurable components including $\mathrm{RF}$ switches and reconfigurable power amplifiers. We also introduce the concept of coarse, medium and fine grain reconfigurable systems and granularity of reconfiguration.

\section{REFERENCES}

[1] Howell, Robert S. et. al. "The Super-Lattice Castellated Field Effect Transistor (SLCFET): A novel high performance Transistor topology ideal for RF switching;" Technical Digest - International Electron Devices Meeting, IEDM, $p$ 11.5.1-11.5.4, February 20, 2015.

[2] Y. Cho et al., "Linear Doherty power amplifier with adaptive bias circuit for average power-tracking," Int'l Microw. Symp, May 2016.

[3] G. Tant et al., "A SOI CMOS reconfigurable output matching network for multimode multiband power amplifiers," Int'l Microw. Symp., May 2015.

[4] H.-S. Chen et al., "A 5.5-GHz multi-mode power amplifier with reconfigurable output matching network," Radio Freq. IC Symp., May 2015.

[5] S. Kang et al., "A multi-mode multi-band reconfigurable power amplifier for $2 \mathrm{G} / 3 \mathrm{G} / 4 \mathrm{G}$ handset applications." IEEE Microw. Wireless Compon. Lett., vol. 25, no. 1, Jan 2015.

[6] D. Gustafsson, C. M. Andersson and C. Fager, "A Modified Doherty Power Amplifier With Extended Bandwidth and Reconfigurable Efficiency," in IEEE Trans. Microw. Theory Techn., vol. 61, no. 1, pp. 533-542, Jan. 2013.

[7] K. Chen and D. Peroulis, "Design of adaptive highly efficient $\mathrm{GaN}$ power amplifier for octave-bandwidth application and dynamic load modulation." IEEE Trans. Microw. Theory Techn., vol. 60, no. 6, June 2012, pp. 1829 1839.

[8] D. J. Shepphard, J. Powell and S. C. Cripps, "An Efficient Broadband Reconfigurable Power Amplifier Using Active Load Modulation," IEEE Microw. Wireless Compon. Lett., vol. 26, no. 6, pp. 443-445, June 2016.

[9] L. Liu, et al., "Hi-Q Tunable Microwave Cavity Resonators and Filters Using SOI-Based RF MEMs Tuners", J. of Micromechanical Syst, vol. 19, no. 4, Aug 2010

[10] I. Reines et al.," Compact Low-Loss Tunable X-Band Bandstop Filter with Miniature RF-MEMS Switches", IEEE Trans. on Microw. Theory and Techn., vol. 19, no. 4, July 2010

[11] L. Kushner et al, "The MATRICs RF-FPGA in 180nm SiGe-on-SOI BiCMOS”, 2015 Radio Frequency IC Symposium

[12] BAA-RQKSE-2015-0006 "Reconfigurable Electronics for Multifunction Agile RF", https://www.fbo.gov/index?s=opportunity\&mode=form\&id $=$ ea9a28f47eaea961deabda8ea1e3b51e\&tab $=$ core \& cview $=$ $\underline{0}, 27$ May 2016 